\title{
ANÁLISIS DEL COMPORTAMIENTO ESPACIO-TEMPORAL INTRADESTINO MEDIANTE GPS: UNA APROXIMACIÓN DE LOS VISITANTES INDEPENDIENTES DE CRUCERO EN LA CIUDAD DE VALÈNCIA
}

\begin{abstract}
Resumen
El presente trabajo tiene como objetivo profundizar en el análisis de los patrones de comportamiento espacio-temporal de los visitantes en destino, una escala de trabajo insuficientemente desarrollada. La investigación aplica un enfoque multimétodo, que combina el uso de GPS con una encuesta, al conocimiento de los patrones espaciales de los cruceristas que visitan la ciudad de Valencia de manera independiente. Los resultados obtenidos evidencian la polarización de los flujos en el centro histórico de la ciudad, dentro de un patrón mayoritario de único nodo, una dinámica que conviene gestionar adecuadamente para prevenir la congestión y poner en valor otros atractivos urbanos. La redistribución de flujos resulta aconsejable pero no es sencilla. Medidas como la redistribución mediante una oferta variada de excursiones plantea dificultades mientras que una mejor adaptación de las líneas de la red de transporte más utilizadas por los cruceristas puede tener efectos más positivos.

Palabras clave: comportamiento espacio-temporal; destinos turísticos inteligentes; gestión turística; seguimiento con GPS; turismo de cruceros.

a Instituto Universitario de Investigaciones Turísticas, Universitat d'Alacant, Carretera San Vicente del Raspeig s/n, 03690, San Vicente del Raspeig - Alicante. sandra.navarro@ua.es https://orcid.org/0000-0001-6651-0538.

b Instituto Universitario de Investigaciones Turísticas, Universitat d'Alacant, Carretera San Vicente del Raspeig s/n, 03690, San Vicente del Raspeig - Alicante. josep.ivar@@ua.es https://orcid.org/0000-0002-9238-2792.

c Departamento de Marketing, Universitat d'Alacant, Carretera San Vicente del Raspeig s/n, 03690, San Vicente del Raspeig - Alicante. ana.casado@ua.es https://orcid.org/0000-0002-9960-0078.
\end{abstract}

Fecha de recepción: 29/1/20. Fecha de aceptación: 17/4/20. 


\title{
ANALYSIS OF INTRA-DESTINATION SPATIAL-TEMPORAL BEHAVIOR USING GPS: AN APPROACH OF INDEPENDENT CRUISE VISITORS IN THE CITY OF VALENCIA
}

\begin{abstract}
The objective of this paper is to delve into the time-space behaviour patterns analysis of visitors at a tourist destination, an insufficiently developed work scale. The research uses a multi-method approach, which combines the use of GPS with a survey, to identify the spatial patterns of cruise passengers who visit the city of Valencia on their own. The results obtained show the polarization of the flows in the historical center of the city, within a majority pattern of a single node, a dynamic that should be adequately managed to prevent congestion and foster other urban attractions. The redistribution of flows is advisable but it is not simple. Measures such as redistribution through a varied offer of excursions pose difficulties while a better adaptation of the transport lines most used by cruise ship visitors can have more positive effects.

KeYwords: cruise tourism; GPS tracking: time-space behaviour; smart tourism destinations; tourism management.
\end{abstract}

\section{INTRODUCCIÓN}

El estudio de los flujos turísticos constituye una línea de investigación esencial de la Geografía del Turismo, sobre todo los flujos origen-destino e interdestinos, ampliamente estudiados desde una perspectiva sistémica (Leiper, 1979; Pearce, 1995). Sin embargo, los flujos intradestino, es decir, los movimientos de los visitantes dentro del destino, han sido menos analizados a pesar de su indudable relevancia para el conocimiento de la actividad turística y para la gestión de la propia actividad y de los espacios receptoresde la misma.

Comprender cómo se comportan los visitantes dentro de un destino es extremadamente relevante para la gestión y planificación del destino ya que permite, entre otras medidas, desarrollar nuevos productos turísticos, mejorar el atractivo del destino y la satisfacción de la demanda,diversificar atractivos turísticos y oferta complementaria, configurar la red de transporte y los servicios básicos, y crear estrategias en aras de mejorar los flujos de visitantes (Dejbakhsh, Arrowsmith y Jackson, 2011; Lew y McKercher, 2006; McKercher y Lau, 2008; MdKhairi e Ismail, 2015; Thornton, Williams y Shaw, 1997). Asimismo, el diagnóstico de comportamiento intradestino permite una gestión más sostenible; optimizando el uso del espacio urbano y evitando conflictos entre residentes y visitantes (Debbage, 1991; Modsching, Kramer, Gretzel y Hagen, 2006; Pearce, 1988; Xia, Zeephongsekul y Packer, 2011).

No obstante, el análisis de los movimientos intradestino se ha visto dificultado según McKercher y Zoltan (2014) por tres razones fundamentales: la necesidad de una mayor precisión en los datos, la escasa confiabilidad de la información aportada por los turistas y la falta de un marco teórico adecuado. Aunque, como señalan los autores mencionados, los nuevos dispositivos tecnológicos permiten superar las dos primeras dificultades, esto no se consigue de forma sencilla y mucho menos automática. Así 
pues, la evolución de las tecnologías de la información y la comunicación (TIC), particularmente las vinculadas a la geolocalización, abren nuevas posibilidades para el estudio de los movimientos intradestino, llegándose a plantear, en el marco de las ciudades o los destinos turísticos inteligentes, la posible gestión en tiempo real de la ciudad/destino (Kitchin, 2015).

Actualmente, la intensidad e interacciones de los flujos urbanos y turísticos en numerosas ciudades, requieren nuevos planteamientos teóricos que ponen el foco en la movilidad (mobilities turn) en los estudios de Geografía del Turismo (Duncan, 2012). En este contexto, la investigación en torno a la movilidad de los cruceristas en destino constituye una línea de trabajo emergente, facilitada por el uso de tecnologías de geolocalización y demandada por sus implicaciones para la gestión turística, especialmente debido a la aparición de fenómenos de saturación turística (overtourism) en algunos destinos urbanos (Brandajs y Russo, 2019). Así pues, el presente trabajo pretende identificar los patrones espacio-temporales de los cruceristas independientes en València, que son visitantes que prefieren explorar el destino por cuenta propia sin contratar una excursión. El objetivo es analizar la incidencia de este tipo de visitantes en el modelo turístico local, determinando los espacios de alta concentración de visitantes y explorando el tiempo medio de visita en ciertos lugares, para poder discernir el perfil de los visitantes independientes de crucero. Para ello, se empleará un enfoque multimétodo de los flujos turísticos intradestino.

\section{EL COMPORTAMIENTO ESPACIO-TEMPORAL EN DESTINO}

El comportamiento intradestino está basado en la individualidad ya que cada sujeto elige cómo moverse y cómo utilizar su tiempo disponible (Pearce, 1988). El análisis de los patrones espacio-temporales ha sido ampliamente abordado en el campo de la movilidad urbana (Delclòs-Alió, Vich y Miralles-Guasch, 2020; Martí, Serrano-Estrada y Nolasco-Cirugeda, 2017; Vich, Marquet y Miralles-Guasch, 2019). En este sentido, las nuevas tecnologías -redes sociales, smartphones, dispositivos GPS, entre otras- han propiciado el incremento de estudios sobre el comportamiento espacio-temporal.

En relación al comportamiento espacio-temporal turístico, diversos estudios se han centrado en analizar cómo la movilidad, accesibilidad y conectividad entre destinos y nodos turísticos afecta al sistema turístico (Biosca et al., 2016; Cejas y Hernández 2017; Coles, Hall y Duval, 2006; Hall, 2005). Tal y como explica Prideaux (2000), la configuración y red de transportes de un destino, tanto para acceder como para desplazarse en el mismo, es de vital importancia para el comportamiento espacio-temporal. No obstante, investigaciones recientes apuntan a la "caminabilidad" del destino, traducción del término anglosajón "walkability” acuñado por Hall y Ram (2019) y Ram y Hall (2018), como otro factor relevante en dicho comportamiento. Este concepto define cuán caminable es el destino sin necesidad de transporte. De esta manera, el sujeto elige "deambular" como forma de movilidad, construyendo un espacio urbano abstracto que determina su comportamiento espacio-temporal y que afecta a la manera de entender la movilidad intradestino.

Por otra parte, el movimiento espacio-temporal turístico también obedece a dos dinámicas interrelacionadas conformadas por los factores personales de empuje (human push factors) y los factores de atracción del destino (physical pull factors) (Lau y McKercher, 2006; Lew y McKercher, 2006). Estudios 
previos han analizado diversos componentes de estos factores tales como la familiaridad con el destino, el tipo de elección de visita, la información previa del destino, el interés inicial en determinados atractivos, la organización de la visita, la duración media, la cantidad de atractivos visitados y las características socio-demográficas del turista (Debbage, 1991; Fakeye y Crompton, 1991; Fennell, 1996; Lau y McKercher, 2006; Lew y McKercher, 2006; McKercher, Wong y Lau, 2006).

Las visitas de los cruceristas también participan de esta interacción entre factores personales y del destino, pero presentan una notable singularidad que viene marcada, en los términos utilizados por Hägerstrand (1970), por la restricción temporal que impone el tiempo de escala. Este hecho afecta de forma considerable el comportamiento intradestino de los visitantes de crucero, sobre todo en los puertos europeos donde se convierten en "cruceristas urbanos", visitantes selectivos y rápidos que consumen una pequeña parte de la ciudad en un tiempo breve (Ashworth y Page, 2011; Navarro-Ruiz, Casado-Díaz e Ivars-Baidal,2019). Por tanto, en el marco de dicha restricción temporal y sus obvias implicaciones espaciales (limitación de la distancia de desplazamiento que afecta a su movilidad, entre otras), cobra relevancia el estudio de Dietvorst (1995) que plantea que la elección de la demanda turística depende de tres elementos interrelacionados: las motivaciones, preferencias y experiencias; las imágenes y estimaciones de oportunidad; y los recursos materiales.

En cuanto a los estudios previos sobre el comportamiento espacio-temporal de los visitantes de crucero, Jaakson (2004) analiza en qué medida los cruceristas se desplazan dentro de una "burbuja turística"en un destino de la costa pacífica mexicana (Zihuatanejo) para identificar diferentes segmentos con un comportamiento espacial diferenciado. Aunque el concepto de burbuja, tan claramente asociado al turismo de masas, resulta interesante en una primera aproximación, conviene matizarlo en el caso de los puertos del Mediterráneo. Así, los trabajos de De Cantis et al. (2016) y Ferrante et al. (2018) que analizan el comportamiento espacial de los cruceristas en Palermo y Dubrovnik, obtienen resultados opuestos a los de Jaakson (2004). Estos estudios concluyen que el promedio de duración de la visita de los visitantes de crucero en los puertos mediterráneos es mayor que en el Caribe, llevando a cabo asimismo dichos cruceristas desplazamientos más amplios por la ciudad portuaria que en el caso del Caribe. Por su parte, Domènech et al. (2020) vinculan el comportamiento espacio-temporal de los cruceristas en Tarragona con el gasto en destino, una relación de indudable relevancia desde la óptica de la planificación urbana, comercial y turística. En esta línea, aunque en un contexto geográfico muy diferente, Lopes y Dredge (2018) analizan el valor para los destinos de las excursiones de los cruceristas en Copenhague. Así pues, se observa el carácter emergente de las investigaciones sobre el comportamiento espacio-temporal de los visitantes de crucero en diferentes puertos de escala, temática a la que el presente estudio pretende contribuir.

El estudio de los patrones espacio-temporales se ha abordado desde dos orientaciones básicas: una basada en los movimientos desde el lugar de localización del alojamiento turístico en forma de anillos concéntricos (Lew y McKercher, 2006), y, otra, más elaborada, estructurada en torno a movimientos lineales que interrelacionan nodos y pueden configurar estructuras reticulares, distinguiéndose básicamente tres tipos de patrones: punto a punto, circulares y patrones complejos (Lew y McKercher, 2006; McKercher y Lau, 2008; Van der Knaap, 1999). Los patrones espaciales modelizan el comportamiento de los visitantes en un destino mediante la agregación de los movimientos individuales (Figura 1). 


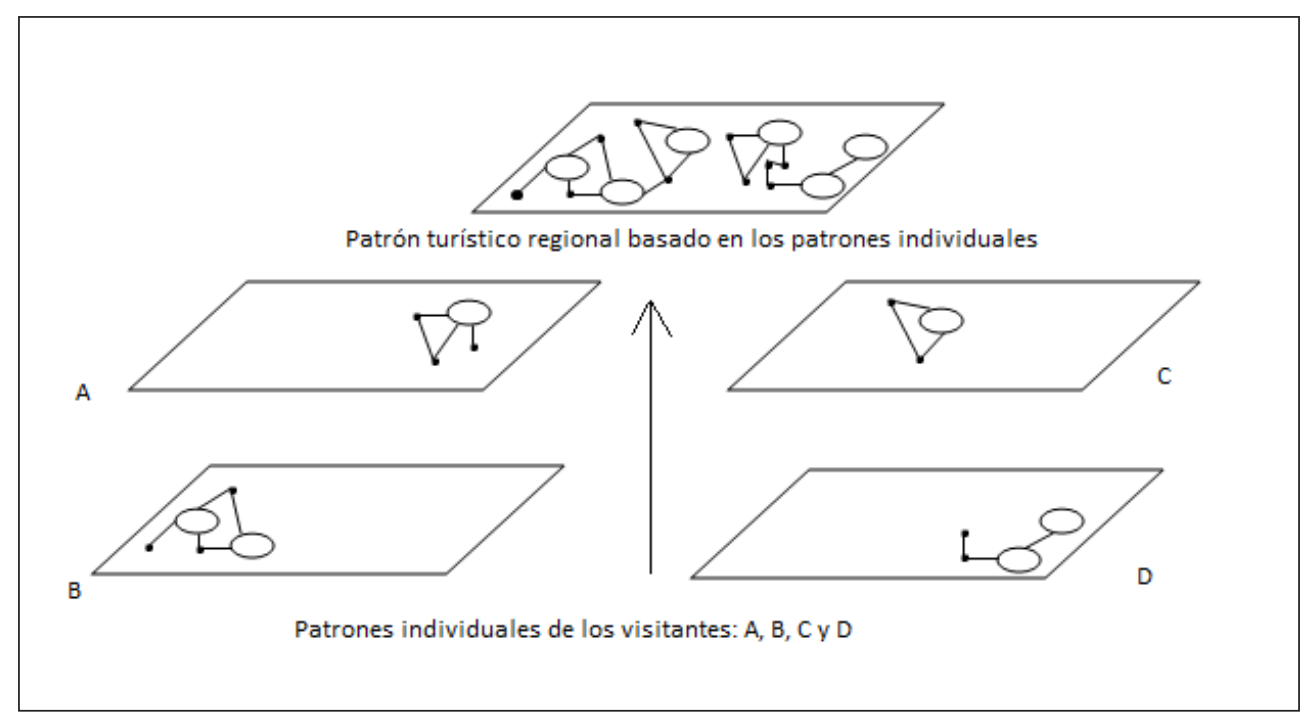

Figura 1. Comportamiento turístico basado en los patrones espacio-temporales individuales. Fuente: Adaptación de Van der Knaap (1997)

Por tanto, los patrones espaciales ofrecen información sobre el grado de intensidad de concentración de visitantes, y sobre la movilidad de los visitantes en función de la accesibilidad a los nodos y la conectividad entre ellos. Dicha agregación es una tarea compleja usando métodos tradicionales (encuestas o cuadernos de viaje), pero se ve facilitada gracias a tecnologías capaces de georreferenciar los desplazamientos de los visitantes (Shoval e Isaacson, 2010).De ahí el uso creciente de estas tecnologías en el análisis de los desplazamientos turísticos y, específicamente, en la identificación de los flujos de cruceristas en destino; convertidos en cruceristas urbanos. Sin embargo, los modelos teóricos de los movimientos lineales no se han tomado como referencia en los estudios empíricos y parecen quedar relegados a modelizaciones abstractas con un valor meramente descriptivo. Así, el presente trabajo supone un intento de adaptación y contraste de los patrones espaciales teóricos con el comportamiento de los cruceristas en Valencia, tomando como referencia los modelos citados (Lew y McKercher, 2006; McKercher y Lau, 2008; Van der Knaap, 1997, 1999).

\section{Metodología PARA LA IDENTIFICACión DE PATRONES ESPACIO-TEMPORALES DE LOS VISITANTES DE CRUCEROS}

\section{València: contexto de estudio}

València ha experimentado una transformación considerable convirtiéndose en un importante destino urbano Europeo (Rausell-Köster et al., 2016), particularmente gracias al desarrollo de nuevas infraestructuras y atractivos turísticos, así como a diferentes estrategias de promoción (Puche-Ruiz y 
Obiol-Menero, 2011; Salom-Carrasco y Pitarch-Garrido, 2017). En este contexto, los Planes Estratégicos de Turismo de València (Fundación Turismo Valencia, 2011, 2017) proponen el turismo de cruceros como un producto emergente para la diversificación turística ya que la ciudad se encuentra ubicada en la región del Mediterráneo Occidental, la segunda región más grande después del Caribe en términos de número de pasajeros de cruceros (MedCruise, 2019).

Así pues, Cervera y García (2016) explican el despegue de la actividad crucerística en València desde su inicio en 2002 en base a diferentes factores tales como la celebración de eventos deportivos de carácter internacional; la mejora de las comunicaciones internas y externas, de las infraestructuras y servicios portuarios, y de la atención al tráfico de cruceros; así como por la liberalización de los horarios comerciales a partir de 2013. En 2018, periodo en el que se desarrolla el presente estudio, la ciudad de València recibió 421.518 cruceristas, situándose como séptimo puerto por volumen de pasajerosen España y cuarto puerto del Mediterráneo español (Puertos del Estado, 2019).

\section{Diseño de la investigación}

Debido a la necesidad de obtener datos de diferente naturaleza, este estudio emplea un enfoque multimétodo para triangular los datos obtenidos (Brewer y Hunter, 2006; Creswell, 2014; Tashakkori y Teddlie, 2003). Este enfoque ha sido utilizado en estudios previos de esta temática (por ejemplo, Beeco et al., 2013; Fennell, 1996; Lau y McKercher, 2006; Li, Yang, Shen y Wu, 2019). Por consiguiente, esta investigación emplea dispositivos GPS y cuestionarios. El uso de los dispositivos GPS se justifica por la fiabilidad de los datos espacio-temporales que ofrecen (Shoval e Isaacson, 2010). En cuanto a la utilización de cuestionarios existe un amplio consenso acerca de su empleo como método para recopilar datos socio-demográficos, de conocimiento del destino, motivaciones o fidelización (Veal, 2006). De este modo, tal y como afirman Li et al. (2019), la fusión de métodos proporciona un conjunto de datos combinados que refleja con una alta precisión y detalle el comportamiento de los visitantes.

El estudio multimétodo se ha realizado con los siguientes instrumentos, un cuestionario inicial para determinar los factores previos a la visita, dispositivos GPS para recopilar los datos sobre el comportamiento espacio-temporal en destino, y un cuestionario final de valoración de la experiencia en destino. Así pues, los encuestadores se aproximaban a los pasajeros independientes dos veces, una vez desembarcaban del barco para visitar la ciudad, y una segunda vez, a la vuelta de la visita. A aquellos pasajeros que aceptaron participar en el estudio, se les realizó el cuestionario inicial sobre su perfil socio-demográfico y factores previos a la visita (familiaridad, información previa, motivaciones, etc.) y, se les entregó el dispositivo GPS. Una vez terminaron la visita y volvían a la terminal, se recogía el dispositivo y se les preguntaba un cuestionario final sobre la experiencia en destino. Se les ofreció un obsequio a todos los participantes.

El modelo de GPS utilizado en este estudio ha sido i-gotU GT-120 USB GPS TravelLogger que registra cada 15 segundos la posición del sujeto midiendo las coordenadas de latitud, longitud, altitud, velocidad y distancia con una precisión de pocos metros y en tiempo real. Cada dispositivo tiene una autonomía de 20 horas lo que asegura su funcionamiento durante toda la escala. Los datos se almace- 
naban automáticamente en el dispositivo para posteriormente descargarlos en un ordenador mediante el software @trip, una vez el visitante hubiese devuelto el GPS. El software @trip permite visualizar los seguimientos en Google Maps y exportarlos a otros formatos ( $g$ p x o kmz) así como su base de datos (a csv), para poder tratar los tracks o itinerarios mediante sistemas de información geográfica.

\section{Datos}

La recopilación de datos se ha llevado a cabo a una muestra de cruceristas en el puerto de València durante los meses de abril y mayo de 2018 (Tabla 1). En el estudio participaron 457 visitantes independientes, de los cuales la muestra final válida fue de 334. Esta disminución se debe a la depuración de datos de los métodos empleados. En lo que respecta a los cuestionarios, se contabilizaron como válidos aquellos individuos que habían contestado tanto el cuestionario inicial como el final. Por parte de los dispositivos GPS, se eliminaron aquellos tracks que tenían saltos temporales provocados por el efecto del cañón urbano (Ferrante et al., 2018).

Tabla 1. Días de la recopilación de los datos. Fuente: Elaboración Propia

\begin{tabular}{|c|c|c|c|}
\hline \multicolumn{2}{|c|}{ Abril 2018} & \multicolumn{2}{|c|}{ Mayo 2018} \\
\hline $04 / 04 / 18$ & Costa Victoria & $04 / 05 / 18$ & MeinSchiff \\
\hline $05 / 0418$ & MSC Divina & $03 / 05 / 18$ & Seabourn \\
\hline $06 / 04 / 18$ & Spirit & $04 / 05 / 18$ & HollandAmerica \\
\hline $09 / 04 / 18$ & Viking & $10 / 05 / 18$ & MSC Divina \\
\hline $11 / 04 / 18$ & MSC Orchestra & $13 / 05 / 18$ & Aida \\
\hline $12 / 04 / 18$ & MSC Divina & $16 / 05 / 18$ & Seven Seas \\
\hline $14 / 04 / 18$ & MeinSchiff & $17 / 05 / 18$ & Costa Pacifica \\
\hline $19 / 04 / 18$ & Queen Victoria & $18 / 05 / 18$ & Jewel of the Seas \\
\hline $20 / 04 / 18$ & Marella & $24 / 05 / 18$ & MSC Divina \\
\hline $21 / 04 / 18$ & Costa Favolosa & $28 / 05 / 18$ & Marella \\
\hline $24 / 04 / 18$ & Seven Seas & $31 / 05 / 18$ & MSC Divina \\
\hline $25 / 04 / 18$ & Marella & & \\
\hline $26 / 04 / 18$ & MSC Divina & & \\
\hline $29 / 04 / 18$ & Aida & & \\
\hline
\end{tabular}

Posteriormente, y para la representación cartográfica del comportamiento espacio-temporal, se ha utilizado el programa libre QGIS 3.4.5 Madeira, mediante el método diseñado por Shoval (2008) y ampliamente utilizado en estos estudios (De Cantis et al. 2016; Shoval e Isaacson, 2010). Se combinaron en un mismo layer las capas vectoriales de puntos de cada individuo, y se creó una cuadrícula o grid de100x100 metros o de 50x50 metros, para contabilizar mediante ambas capas tanto los individuos 
que estaban en cada una de las celdas de la cuadrícula, como el tiempo de cada individuo para obtener el promedio de tiempo por celda.

Finalmente, los datos recopilados mediante los cuestionarios fueron grabados en el programa estadístico SPSS versión 24 para poder llevar a cabo un análisis descriptivo; caracterizando el perfil de la demanda de cruceristas en Valencia.

\section{Resultados}

En primer lugar, los patrones de comportamiento de los visitantes independientes se diferenciaron según su relación con el número de nodos visitados (un solo nodo o múltiples nodos), entendiendo los nodos turísticos como las áreas, distritos o clusters que agrupan varios atractivos turísticos (Dredge, 1999). Para poder discernir las concentraciones específicas en cada nodo turístico, la delimitación en detalle se ha realizado a partir de un análisis de intensidad del número de visitantes y tiempo medio por celda. Las nubes de puntos de la muestra estudiada muestran que los visitantes de crucero tienden a visitar cuatro nodos turísticos esencialmente: (1) Bioparc, (2) centro histórico donde se encuentran la mayoría de los atractivos culturales, (3) Ciudad de las Artes y las Ciencias, un complejo vanguardista y, (4) el área de la Marina Real y paseo marítimo (aunque el primer y cuarto nodo con una menor intensidad).

Tal y como se aprecia en la Tabla 2, el 62,9\% de loscruceristas independientes prefieren visitar un único nodo, mientras que el 37,1\% opta por visitar varios nodos turísticos. Por tanto, dado que para la muestra analizada representa el patrón más frecuente, se decide analizar en profundidad el comportamiento espacio-temporal en un único nodo, descartando del estudio los cruceristas independientes que visitaron múltiples nodos.

Tabla 2. Distribución de visitantes independientes por nodo turístico especifico visitado.

Fuente: Elaboración propia

\begin{tabular}{|l|l|l|l|}
\hline & Frecuencia & $\begin{array}{c}\% \text { sobre el total } \\
\text { de cruceristas } \\
\text { independientes }\end{array}$ & $\begin{array}{c}\text { \% sobre el } \\
\text { subsegmento UN }\end{array}$ \\
\hline $\begin{array}{l}\text { Cruceristas independientes que visitan un único nodo } \\
\text { (UN): Nodo visitado }\end{array}$ & & & \\
\hline CAC & 26 & $7,8 \%$ & $12,4 \%$ \\
\hline Centro Histórico & 177 & $53,0 \%$ & $84,3 \%$ \\
\hline Paseo marítimo-Marina Real & 6 & $1,8 \%$ & $2,9 \%$ \\
\hline Bioparc & 1 & $0,3 \%$ & $0,5 \%$ \\
\hline \multicolumn{1}{|c|}{ Subtotal } & 210 & $62,9 \%$ & $100,0 \%$ \\
\hline $\begin{array}{l}\text { Cruceristas independientes que visitan múltiples nodos } \\
\text { (MN) }\end{array}$ & 124 & $37,1 \%$ & \\
\hline Total muestra & 334 & $100,0 \%$ & \\
\hline
\end{tabular}


Respecto a la representación gráfica de dichos patrones espacio-temporales, en los mapas 1 y 2 se muestra en gradación de color las concentraciones de visitantes clasificadas por intervalo de Jenks (de verde oscuro- menos concentración de visitantes- a rojo - más concentración), mientras que la altura de las columnas muestra el promedio de tiempo en cada celda. El Mapa 1 recoge la presencia de visitantes en celdas de 100×100 metros, mientras que la tabla de los atractivos turísticos, ubicada a pie del mapa, está ordenada por el volumen de afluencia, de mayor a menor, es decir, los cinco primeros atractivos se contabilizaron como los más visitados.

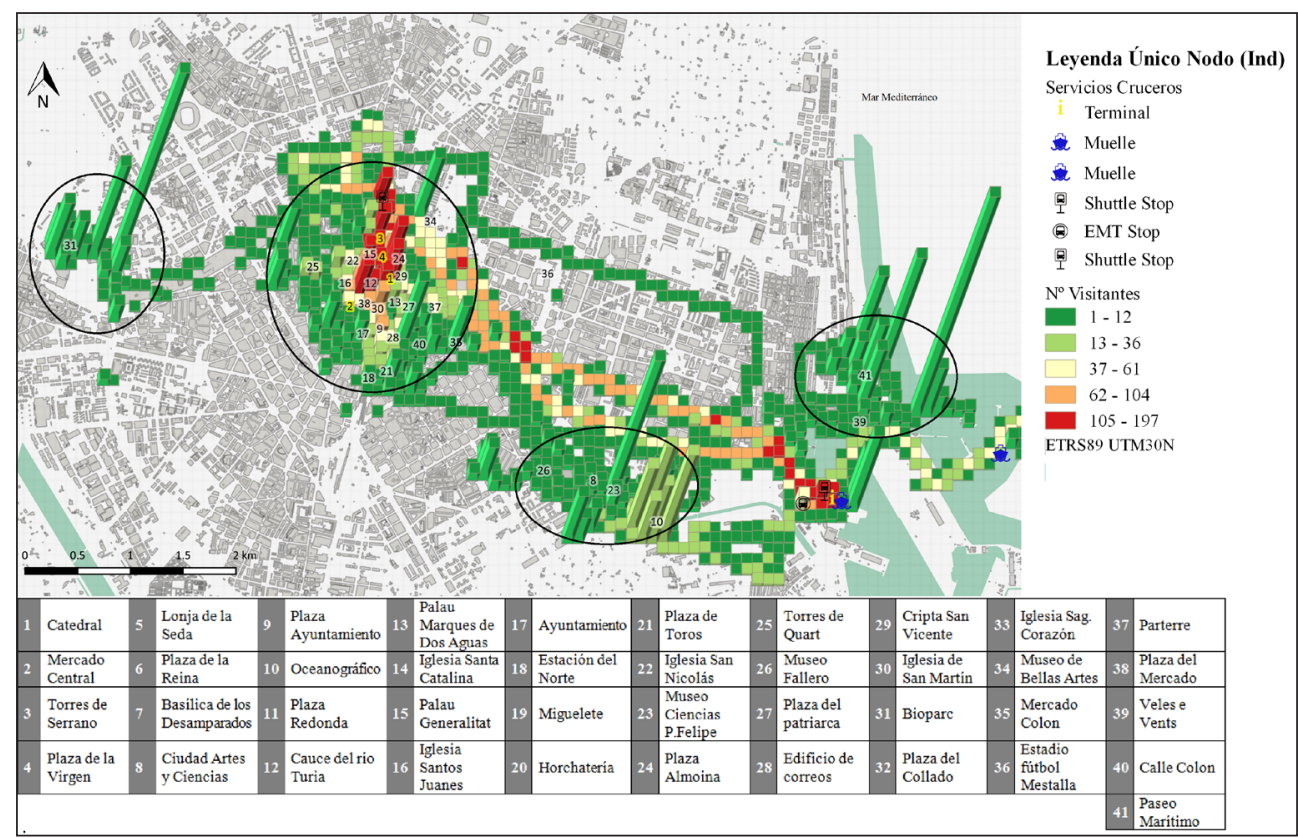

Mapa 1. Comportamiento espacio-temporal de visitantes de crucero independientes en un único nodo. Fuente: Elaboración propia

En primer lugar, el mapa refleja una acusada concentración en el centro histórico, mientras que en el resto de nodos la concentración es baja (de 1 a 36 personas, lo que supone hasta un 17,5\% de la muestra). Aún así, el promedio de tiempo en Bioparc, paseo marítimo-Marina Real y CAC es mayor que en el centro histórico. En segundo lugar, el número de visitantes independientes que visitan únicamente la CAC representa el $12,4 \%$ de los independientes que se desplazan a un único nodo. Este porcentaje tan bajo puede deberse a la red de transportes, puesto que no existe parada de shuttle en dicho nodo turístico, y por consiguiente desde la terminal de cruceros únicamente se puede acceder a la CAC a pie $(2,5 \mathrm{~km})$, en taxi o transporte público (una línea). En cuanto a los atractivos visitados, dicho segmento elige el Oceanográfico como atractivo principal, cuya media de visita alcanza las 3 ho- 
ras y media. En el resto de edificiosla ratio de presencia de visitantes es menor pero la media temporal es alta: el Museo Príncipe Felipe tiene una visita media de hora y media, y el resto del complejo de 2 horas; el centro comercial de El Saler de 50 minutos; y el cauce del río Turia (que tan solo contabiliza a un pasajero) de una hora y 10 minutos. En tercer lugar, el nodo del paseo marítimo-Marina Real presenta una concentración baja (2,7\% de los visitantes independientes de único nodo). No obstante, en este nodo turístico es donde se encuentran los mayores promedios de tiempo por celda. En el paseo marítimo, la media de tiempo es de 2 horas y cuarto y, en la Playa de las Arenas se alcanza una media de una hora y 10 minutos, en contraposición, en la Marina Real -Veles e Vents y sus alrededores- se obtiene una media de 30 minutos. Por último, y en relación con la columna más alta, ésta corresponde a una única persona que realizó la actividad náutica de jetsky durante 2 horas.

El Mapa 2, en celdas de 50x50 metros, simboliza un eje rojo continuo que representa las ratios de concentración de visitantes más elevados.

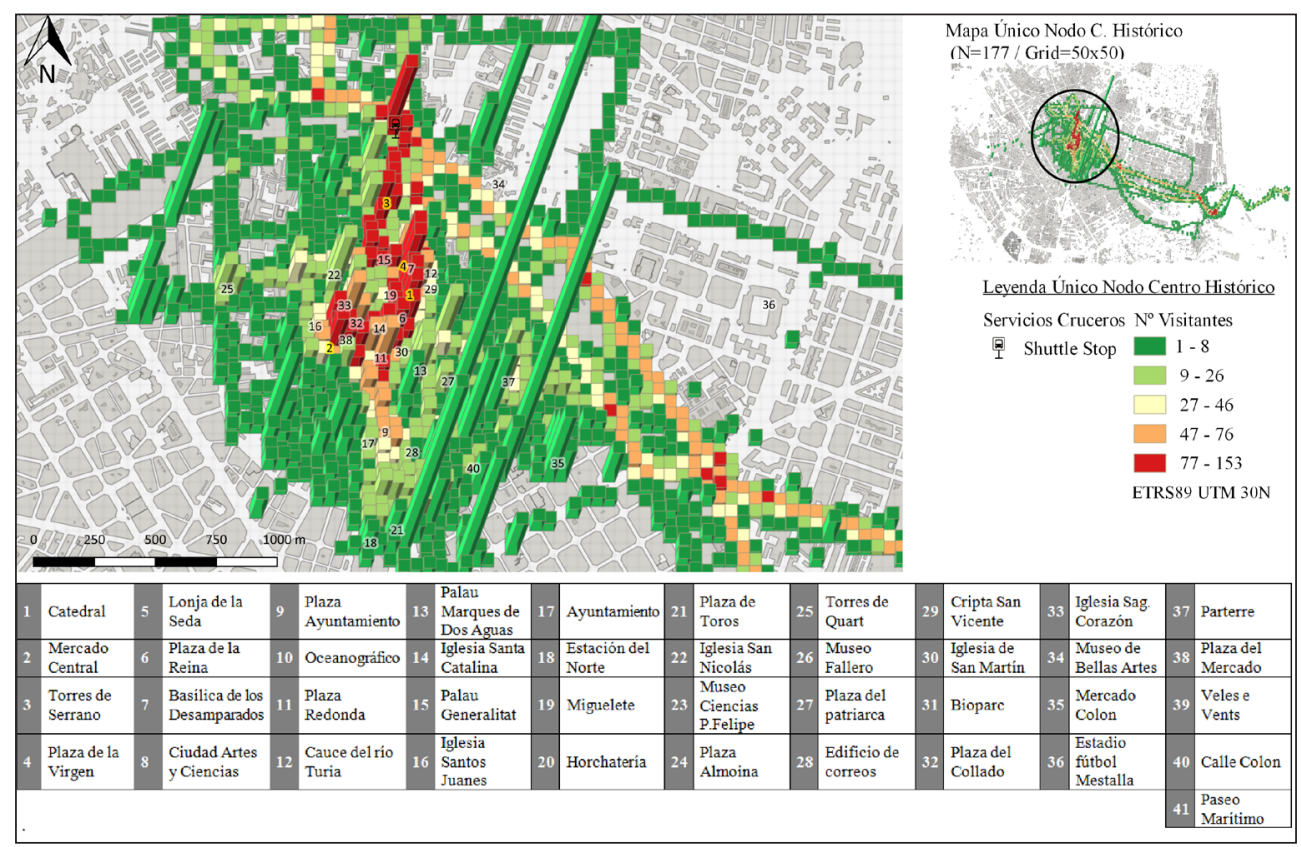

Mapa 2. Comportamiento espacio-temporal de visitantes independientes en centro histórico como único nodo. Fuente: Elaboración propia 
Los altos ratios de presencia en el centro histórico (Mapa 2) pueden tener su explicación en la ubicación de la parada del autobús lanzadera de las navieras (shuttle), localizada enfrente de las Torres de Serrano. Dicho recorrido comienza en la parada del shuttle, pasa después por las Torres de Serrano hasta la Calle Caballeros, se dirige a la Plaza de la Virgen, continúa por Plaza de la Reina y Calle San Vicente, gira por Calle María Cristina y llega al Mercado Central. Asimismo, con una intensidad media de afluencia, los visitantes se desplazan a la Plaza del Ayuntamiento, la Lonja de la Seda o visitan la Plaza Redonda. Mientras que el resto de recursos turísticos del centro obtiene unas ratios de concentración de afluencia baja, destacando la Estación del Norte, Plaza de Toros y Torres de Quart. Respecto al promedio de tiempo en celda, los resultados difieren del resto de nodos ya que las medias no alcanzan concentraciones de tiempo tan altas: en la Plaza de la Reina el tiempo medio es de 35 minutos, la visita a la Catedral consta de un promedio de 30 minutos, seguida por la Plaza de la Virgen y el Mercado Central que contabilizan una media de 25 minutos. Mientras que las Torres de Serrano y Quart, Estación del Norte o Plaza Redonda obtienen medias de 10 minutos de visita.

En relación a los resultados obtenidos de las encuestas (Tabla 3), desde el punto de vista del perfil de los visitantes, el tamaño de las submuestras sólo permite un análisis exploratorio de carácter descriptivo carente de significación estadística de los datos procedentes de los cuestionarios. 
Tabla 3. Perfil de los visitantes de crucero según el nodo turístico visitado.

Fuente: Elaboración Propia

\begin{tabular}{|c|c|c|c|c|c|c|}
\hline & Factores & Ítems & $\begin{array}{l}\text { Total muestra } \\
\qquad(\mathrm{N}=334) \\
\text { Porcentajes }\end{array}$ & $\begin{array}{l}\text { Indep. UN } \\
(\mathrm{n}=210) \\
\text { Porcentajes }\end{array}$ & $\begin{array}{c}\text { UN } \\
\text { Centro(n=177) } \\
\text { Porcentajes }\end{array}$ & $\begin{array}{l}\text { UN CAC } \\
\quad(n=26) \\
\text { Porcentajes }\end{array}$ \\
\hline & Sexo & $\begin{array}{l}\text { Hombre } \\
\text { Mujer }\end{array}$ & $\begin{array}{l}37,4 \\
62,6\end{array}$ & $\begin{array}{l}36,2 \\
63,8\end{array}$ & $\begin{array}{l}33,9 \\
66,1\end{array}$ & $\begin{array}{l}50 \\
50\end{array}$ \\
\hline & Edad & $\begin{array}{l}18-25 \\
26-35 \\
36-45 \\
46-55 \\
56-65 \\
66 \text { o más }\end{array}$ & $\begin{array}{l}2,4 \\
8,7 \\
15,9 \\
21,6 \\
27,2 \\
24,3\end{array}$ & $\begin{array}{l}3,3 \\
7,1 \\
11,4 \\
21 \\
29 \\
28,1\end{array}$ & $\begin{array}{l}2,3 \\
4,5 \\
10,7 \\
19,8 \\
31,6 \\
31,1\end{array}$ & $\begin{array}{l}11,5 \\
11,5 \\
19,2 \\
26,9 \\
19,2 \\
11,5\end{array}$ \\
\hline & Nivel de estudios & $\begin{array}{l}\text { Básica / Primaria } \\
\text { Secundaria } \\
\text { Bachiller / FP } \\
\text { Universitarios }\end{array}$ & $\begin{array}{l}2,1 \\
10,2 \\
37,7 \\
50\end{array}$ & $\begin{array}{l}2,9 \\
11,4 \\
39 \\
46,7\end{array}$ & $\begin{array}{l}2,8 \\
11,3 \\
40,1 \\
45,8\end{array}$ & $\begin{array}{l}3,8 \\
7,7 \\
34,6 \\
53,8\end{array}$ \\
\hline 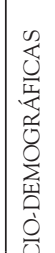 & Situación empleo & $\begin{array}{l}\text { Empleo en activo } \\
\text { Jubilado/Pensionista } \\
\text { Desempleado } \\
\text { Estudiante } \\
\text { Labores del hogar } \\
\text { Otros }\end{array}$ & $\begin{array}{l}57,5 \\
34,4 \\
0,3 \\
1,5 \\
0,6 \\
5,7\end{array}$ & $\begin{array}{l}51,4 \\
41 \\
0,5 \\
1 \\
0 \\
6,2\end{array}$ & $\begin{array}{l}50,3 \\
45,2 \\
0,6 \\
0 \\
0 \\
4\end{array}$ & $\begin{array}{l}53,8 \\
19,2 \\
0 \\
7,7 \\
0 \\
19,2\end{array}$ \\
\hline 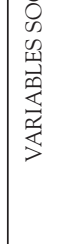 & $\begin{array}{l}\text { Ingreso neto } \\
\text { mensual unidad } \\
\text { familiar }\end{array}$ & $\begin{array}{l}\text { Menos de } 1.000 € \\
1.000 €-2.000 € \\
2.001-3.000 € \\
3.001-4.000 € \\
\text { Más de } 4.000 € \\
\text { NS / NC }\end{array}$ & $\begin{array}{l}1,2 \\
12 \\
13,8 \\
16,2 \\
36,8 \\
20,1\end{array}$ & $\begin{array}{l}1 \\
15,7 \\
12,9 \\
16,2 \\
36,2 \\
18,1\end{array}$ & $\begin{array}{l}1,1 \\
15,8 \\
12,4 \\
15,3 \\
37,9 \\
17,5\end{array}$ & $\begin{array}{l}0 \\
19,2 \\
11,5 \\
15,4 \\
26,9 \\
26,9\end{array}$ \\
\hline & Región residencia & $\begin{array}{l}\text { Europa } \\
\text { Norte América } \\
\text { Latino América } \\
\text { Asia-Pacífico } \\
\text { Otros }\end{array}$ & $\begin{array}{l}81,7 \\
9,3 \\
2,4 \\
4,9 \\
1,7\end{array}$ & $\begin{array}{l}84,3 \\
9,9 \\
2,9 \\
2,9 \\
0\end{array}$ & $\begin{array}{l}77,4 \\
14,7 \\
2,8 \\
5,1 \\
0\end{array}$ & $\begin{array}{l}92,3 \\
7,7 \\
0 \\
0 \\
0\end{array}$ \\
\hline & $\begin{array}{l}\text { Composición grupo } \\
\text { visita }\end{array}$ & $\begin{array}{l}\text { Solo/a } \\
\text { Con pareja } \\
\text { Con amigos/as } \\
\text { Con familia } \\
\text { Con pareja y amigos } \\
\text { Con familia y amigos }\end{array}$ & $\begin{array}{l}1,5 \\
56,3 \\
6,6 \\
28,7 \\
5,7 \\
1,2\end{array}$ & $\begin{array}{l}1,4 \\
58,6 \\
8,1 \\
26,2 \\
5,2 \\
0,5\end{array}$ & $\begin{array}{l}1,1 \\
59,9 \\
9 \\
9 \\
23,2 \\
6,2\end{array}$ & $\begin{array}{l}3,8 \\
57,7 \\
3,8 \\
34,6 \\
0 \\
0\end{array}$ \\
\hline
\end{tabular}




\begin{tabular}{|c|c|c|c|c|c|c|}
\hline & Factores & Ítems & $\begin{array}{c}\text { Total muestra } \\
\quad(\mathrm{N}=334) \\
\text { Porcentajes }\end{array}$ & $\begin{array}{l}\text { Indep. UN } \\
\quad(\mathrm{n}=210) \\
\text { Porcentajes }\end{array}$ & $\begin{array}{c}\text { UN } \\
\text { Centro(n=177) } \\
\text { Porcentajes }\end{array}$ & $\begin{array}{l}\text { UN CAC } \\
\quad(n=26) \\
\text { Porcentajes }\end{array}$ \\
\hline \multirow{6}{*}{ 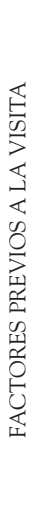 } & Viaje en crucero & $\begin{array}{l}\text { Noveles } \\
\text { Repetidores }\end{array}$ & $\begin{array}{l}24,6 \\
75,4\end{array}$ & $\begin{array}{l}25,7 \\
74,3\end{array}$ & $\begin{array}{l}27,1 \\
72,9\end{array}$ & $\begin{array}{l}11,5 \\
88,5\end{array}$ \\
\hline & $\begin{array}{l}\text { Conocimiento de } \\
\text { Valencia }\end{array}$ & $\begin{array}{l}\text { Noveles } \\
\text { Repetidores }\end{array}$ & $\begin{array}{l}76,3 \\
23,7\end{array}$ & $\begin{array}{l}72,4 \\
27,6\end{array}$ & $\begin{array}{l}75,7 \\
24,3\end{array}$ & $\begin{array}{l}46,2 \\
53,8\end{array}$ \\
\hline & $\begin{array}{l}\text { Importancia } \\
\text { Valencia }\end{array}$ & $\begin{array}{l}\text { Factor importante } \\
\text { Sin importancia }\end{array}$ & $\begin{array}{l}46,1 \\
53,9\end{array}$ & $\begin{array}{l}45,7 \\
54,3\end{array}$ & $\begin{array}{l}47,5 \\
52,5\end{array}$ & $\begin{array}{l}46,2 \\
53,8\end{array}$ \\
\hline & $\begin{array}{l}\text { Información previa } \\
\text { destino }\end{array}$ & $\begin{array}{l}\text { Búsqueda de información } \\
\text { Sin información }\end{array}$ & $\begin{array}{l}80,2 \\
19,8\end{array}$ & $\begin{array}{l}80 \\
20\end{array}$ & $\begin{array}{l}79,7 \\
20,3\end{array}$ & $\begin{array}{l}80,8 \\
19,2\end{array}$ \\
\hline & $\begin{array}{l}\text { Organización } \\
\text { tiempo disponible }\end{array}$ & $\begin{array}{l}\text { Organización del tiempo } \\
\text { Sin organización }\end{array}$ & $\begin{array}{l}45,5 \\
54,5\end{array}$ & $\begin{array}{l}44,8 \\
55,2\end{array}$ & $\begin{array}{l}41,2 \\
58,8\end{array}$ & $\begin{array}{l}76,9 \\
23,1\end{array}$ \\
\hline & $\begin{array}{l}\text { Interés especial en } \\
\text { atractivos }\end{array}$ & $\begin{array}{l}\text { Interés especial } \\
\text { Sin interés }\end{array}$ & $\begin{array}{l}59,7 \\
40,3\end{array}$ & $\begin{array}{l}55,7 \\
44,3\end{array}$ & $\begin{array}{l}50,8 \\
49,2\end{array}$ & $\begin{array}{l}84,6 \\
15,4\end{array}$ \\
\hline
\end{tabular}

En síntesis, se aprecia una mayor representación en los visitantes al centro histórico de personas de origen europeo (92,3\%), frente a un 77,4\% europeos y 14,7\% norteamericanos y canadienses que visitan la CAC. En relación a la edad, hay mayor presencia de personasde mayor edad que visitan el centro histórico (el 31,1\% es mayor de 66 años) frente al 11,5\% de los visitantes del rango de esta edad que se desplaza a la CAC. Por último, es reseñable que en ambos casos más del 50\% visita el nodo turístico en pareja, pero en la CAC el 34,6\% son familias frente a un 9\% de familias que visita el centro histórico. Por otra parte, el $75,7 \%$ de los visitantes del centro es la primera vez que viajan a Valencia frente al $46,2 \%$ de los que visitan la CAC. Otra cuestión remarcable de los factores previos a la visita es que el $49,2 \%$ de los que estuvieron en el centro declararon un interés especial previo por algún atractivo de la ciudad, porcentaje que se eleva al $84,6 \%$ para los visitantes de la CAC.

Finalmente, y en cuanto a la variable temporal, sólo el 41,2\% de los cruceristas del centro histórico organizaron su tiempo disponible para la visita comparado con el 76,9\% de los visitantes de la CAC que sí planificaron su tiempo. Del mismo modo, y respecto a la duración total de la visita, los visitantes que acudieron a la CAC y que ocuparon más de 4 horas de visita suponen el 84,5\% frente al 52\% de los que acudieron al centro histórico. Esto supone que el $48 \%$ de los visitantes del centro histórico estuvieron menos de 4 horas visitando dicho nodo turístico.

\section{Discusión Y CONCLUSIONES}

El conocimiento del comportamiento espacio-temporal de los visitantes de crucero dentro de los destinos es incuestionablemente relevante ya que consumen el destino, sus atractivos y sus servicios en un período de tiempo limitado. De la investigación realizada se derivan diferentes aspectos relacionados con la práctica espacio-temporal de los cruceristas y su influencia en el modelo turístico urbano, así como cuestiones metodológicas relativas al estudio de los flujos de visitantes en destino. 
Respecto a la influencia en el modelo turístico urbano, la dinámica espacio-temporal de las visitas de cruceristas refuerza la concentración de flujos en los atractivos urbanos más visitados y, por consiguiente, la centralidad de los principales nodos turísticos urbanos o, como en el caso de València, de un único nodo. Es el comportamiento característico de los puertos de escala concebidos como "agujero negro" (blackhole), donde las visitas se centran de manera generalizada en los atractivos icónicos o primarios (Gui y Russo, 2011; Rodrigue y Notteboom, 2013).

La visita al centro histórico como un único nodoconstituye el patrón mayoritario, corroborado por otros estudios (Agencia Valenciana del Turismo, 2015). Este hecho enfatiza la caracterización de los pasajeros de crucero como cruceristas urbanos que consumen una parte de la ciudad histórica en un tiempo limitado. Por otra parte, los resultados revelan que los nuevos atractivos turísticos de la ciudad, fundamentalmente la CAC, promovida por las Administraciones Públicas como gran apuesta estratégica (Puche-Ruiz y Obiol-Menero, 2011; Salom-Carrasco y Pitarch-Garrido, 2017), demuestran una capacidad limitada de redistribución de flujos, al menos para la demanda de cruceros.

Las visitas de los cruceristas participan de un tipo de mirada colectiva (Urry y Larsen, 2011) en la que la concentración de un número considerable de personas indica los lugares que merece la pena visitar, una perspectiva que entronca con prácticas de consumo fordistas. La polarización de flujos intraurbanos favorecida por las visitas de los cruceristastiene implicaciones negativas para el modelo turístico local ya que favorece la congestión de los recursos más visitados, con una posible degradación de la experiencia turística e impactos negativos para los residentes, además de infrautilizar otros atractivos de la localidad. Esta dinámica está claramente relacionada con los procesos de saturación turística en espacios públicos urbanos, que todavía no son constatables en la ciudad de València como un problema grave, pero sí constituyen una amenaza que conviene prevenir (Del Chiappa, Lorenzo-Romero and Gallarza, 2018).

Los procesos de overtourism, de carácter multicausal y variables según cada contexto territorial, superan los objetivos de este trabajo, pero sí resulta interesante plantearse en qué medida la redistribución de los flujos de los visitantes de cruceros puede contribuir a prevenir la congestión turística. La redistribución es una solución frecuentemente planteada desde la óptica teórica y menos habitual en la práctica (Navarro-Ruiz et al. 2019). En esencia, está relacionada con los movimientos intradestino y, por tanto, condicionada por los factores de demanda y de atractivo del destino, a los que hay que añadir los intereses de las compañías navieras. Las navieras se nutren de un modelo de negocioque exige ingresos complementariosa la venta del viaje en crucero, como los derivados de las excursiones (Vogel, 2011).

Indudablemente, las excursiones son una oportunidad para redistribuir flujos y ampliar el hinterland de los puertos de escala, es decir, los desplazamientos más allá de la ciudad portuaria, además de tener un efecto positivo en la experiencia turística en la ciudad de Valencia (Sanz-Blas y Buzova, 2016). No obstante, en el caso de Barcelona y València, la oferta de excursiones promueve los atractivos más frecuentados (Navarro-Ruiz et al., 2019) y la demanda contrata escasamente las excursiones fuera de los lugares turísticos centrales, de manera que la polarización de los flujos se ve favorecida tantodesde el punto de vista de la oferta comode la demanda. Por tanto, la redistribución de los flujos constituye 
uno de los principales retos de gestión del turismo de cruceros en el que hay que incluir la perspectiva de valor para los destinos (Lopes y Dredge, 2017).

Otra medida que se deriva de los resultados de este estudio parece más eficaz, sobre todo para los cruceristas independientes. El comportamiento intradestino está claramente influido por la red de transporte y sus paradas. En el caso de Valencia se observa que únicamente existe parada de shuttle en el centro histórico, enfrente de las Torres de Serrano, la cual se convierte en el punto de partida del eje de mayor intensidad de visitantes de crucero, por lo que una ampliación del número de paradas del shuttle podría redirigir los flujos para evitar la concentración y poner en valor otros atractivos urbanos, tanto del nodo del centro histórico como de otros nodos de la ciudad.

De este modo, se considera que una futura línea de investigación muy relevante sería analizar si la estrategia propuestade redistribución de flujos mediante la inclusión de nuevas paradas de shuttle provoca impactos positivos o negativos en el destino. Asimismo, sería interesante comprobar la velocidad media de los desplazamientos entre los nodos y dentro de los nodos (entre atractivos) para discernir cuáles son espacios de visita rápida y lenta. Por otra parte, otro futuro estudio radicaría en comparar las diferencias socio-demográficas en función de grupos con una duración media de la visita similar, así como comparar el comportamiento espacio-temporal intradestino de los visitantes independientes y los visitantes guiados. Por último, en relación a las recientes investigaciones sobre los destinos turísticos inteligentes, sería conveniente comprobar si este nuevo paradigma de planificación y gestión es capaz de medir en tiempo real el comportamiento espacio-temporal, y qué efectos tienen las decisiones que se toman para paliar posibles impactos.

Desde el punto de vista metodológico, la utilización de GPS presenta ventajas sobre los métodos tradicionales, pero también limitaciones (Donaire et al., 2015; Grinberger et al., 2014; Ivars et al., 2016; Shoval e Isaacson, 2010). En el presente estudio se han conseguido reducir los errores más comunes en el uso de dispositivos GPS, en particular los puntos desubicados o perdidos, gracias a la buena señal satélite del destino, la sencillez del uso del dispositivo para un perfil de demanda como el visitante de cruceros, la fácil identificación de los puntos en los espacios interiores y el uso de métodos complementarios que permiten refinar la información georreferenciada (cuestionario y cuaderno de viaje). Sin embargo, estas mejoras se producen mediante un esfuerzo considerable en el trabajo de campo y en la depuración de los datos.

La relevancia del estudio de los flujos de visitantes desde el punto de vista de la investigación resulta incuestionable pero su generalización como herramienta de gestión presenta mayores dificultades. Los métodos y las tecnologías asociadas son diversos de acuerdo con los objetivos de cada proyecto relacionado con los movimientos de los visitantes en destino o la identificación de los espacios más frecuentados: uso de GPS, geolocalización de usuarios de telefonía móvil, despliegue de una red de sensores, monitorización de redes sociales, uso de aplicaciones móviles o conexión a redes wifi gratuitas, entre otras. Muchas de estas soluciones se enmarcan en el nuevo enfoque de las ciudades y/o destinos inteligentes, un planteamiento interesante por su enfoque sistémico y de coordinación de las fuentes de información pero que debe responder a las necesidades de cada destino y no a los intereses de las 
empresas tecnológicas, respetar la privacidad de los ciudadanos y turistas, garantizar la seguridad de la información y evitar nuevas dependencias de carácter tecnológico.

\section{Agradecimientos}

Esta investigación se ha desarrollado en el marco del Proyecto de investigación "Análisis de procesos de planificación aplicados a ciudades y destinos turísticos inteligentes. Balance y propuesta metodológica para espacios turísticos: Smart Tourism Planning”. Proyecto CSO2017-82592-R del Programa Estatal de I+D+i del Ministerio de Economía, Industria y Competitividad (2018-2020). [UAFPU2016-014]

\section{Referencias}

Agencia Valenciana de Turismo (2015): Perfil del crucerista extranjero que realiza escala en los puertos de la Comunitat Valenciana, Estudio elaborado por Gfk, url:<http://www.turisme.gva.es/turisme/es/files/ pdf/estadistiquesdeturisme/estudios_producto/Cruceros_agosto2016.pdf > (consulta: 12/2/2019).

Ashworth, G. y Page, S. J. (2011): Urban tourism research: Recent progress and current paradoxes, Tourism Management, 32 (1), 1-15, doi: 10.1016/j.tourman.2010.02.002

Beeco, J. A., Huang, W.J., Hallo, J. C., Norman, W. C., McGehee, N. G., McGee, J., \& Goetcheus, C. (2013): GPS Tracking of Travel Routes of Wanderers and Planners. Tourism Geographies, 15(3), 551-573.

Biosca, S. A. O., Escobedo, J. A. S., \& Martínez, M. D. L. S. (2016): Planificación de rutas turísticas para autobús a través de indicadores de accesibilidad integral y de dotación de bienes materiales e inmateriales. Revista Transporte y Territorio, (14), 144-166.

Brandajs, F. y Russo, A. P. (2019): Whose is that square? Cruise tourists' mobilities and negotiation for public space in Barcelona, Applied Mobilities, doi: 10.1080/23800127.2019.1576257

Brewer, J., \& Hunter, A. (2006): Foundations of multimethod research: Synthesizing styles. SAGE.

Cejas, F. R. R. M., \& Hernández, P. M. (2017): La movilidad turística en la Isla de Lanzarote: el diseño de una ruta para un autobús turístico. International Journal of Scientific Management and Tourism, 3(3), 459-477.

Cervera, A. y G. García (2016): Pasado, presente y futuro del turismo de cruceros: el caso de Valencia, en Boira, J.V. (coord.), Turismo y ciudad. Reflexiones en torno a València, Valencia, Universitat de València, 41-52.

Coles, T., Hall, C. M., \&Duval, D. T. (2006): Tourism and post-disciplinary enquiry. Current Issues in Tourism, 9(4-5), 293-319.

Creswell, J. (2014): The selection of a research approach. In J. W. Creswell (Ed.), Research design: qualitative, quantitative, and mixed methods approaches. (pp. 3-23). SAGE. 
De Cantis, S., Ferrante, M., Kahani, A. and Shoval, N. (2016): Cruise passengers' behavior at the destination: Investigation using GPS technology, Tourism Management, 52, 133-150, doi:10.1016/j. tourman.2015.06.018

Debbage, K. G. (1991): Spatial behavior in a Bahamian resort. Annals of Tourism Research, 18(2), 251-268.

Del Chiappa, G., Lorenzo-Romero, C., \& Gallarza, M. (2018): Host community perceptions of cruise tourism in a homeport: A cluster analysis. Journal of Destination Marketing $\mathcal{E}$ Management, 7, 170-181.

Delclòs-Alió, X., Vich, G., \&Miralles-Guasch, C. (2020): The relationship between Mediterranean built environment and outdoor physical activity: evidence from GPS and accelerometer data among young adults in Barcelona. Landscape Research, 00(00), 1-14. https://doi.org/10.1080/0142 6397.2019.1702937

Dejbakhsh, S., Arrowsmith, C., \& Jackson, M. (2011): Cultural Influence on Spatial Behaviour. Tourism Geographies: An International Journal of Tourism Space, Place and Environment, 13(1), 91-111.

Dietvorst, A. (1995): Tourist behaviour and the importance of time-space analysis, en G. J. Asworth y A. Dietvorst (eds.), Tourism and Spatial Transformations: Implications for Policy and Planning, Wallingford: CABI, 163-181.

Domènech, A., Gutiérrez, A. y Anton Clavé, S. (2020): Cruise Passengers' Spatial Behaviour and Expenditure Levels at Destination, Tourism Planning and Development, 17(1), 17-36. doi: 10.1080/21568316.2019.1566169

Donaire, J.A., Galí, N. y Royo-Vela, M. (2015): El Uso de GPS Para El Análisis Del Comportamiento Espacio-Temporal de Los Turistas. Pre-Test En El Valle de Boí. Cuadernos de Turismo, 35, 117-131.

Dredge, D. (1999): Destination place planning and design. Annals of Tourism Research, 26(4), 772-791.

Duncan, T. (2012): The 'Mobilities Turn' and the Geography of Tourism, en Wilson, J. (ed.), The Routledge Handbook of Tourism Geographies, Oxon: Routledge, 113-119.

Fakeye, P. C., \& Crompton, J. L. (1991): Image Differences between Prospective, First-Time, and Repeat Visitors to the Lower Rio Grande Valley. Journal of Travel Research, 30 (2), 10-16.

Fennell, D. A. (1996): A tourist space-time budget in the Shetland Islands. Annals of Tourism Research, 23(4), 811-829.

Ferrante, M., De Cantis, S. y Shoval, N. (2018): A general framework for collecting and analysing the tracking data of cruise passengers at the destination, Current Issues in Tourism, 21 (12), 1426-1451, doi: 10.1080/13683500.2016.1194813

Fundación Turismo Valencia (2011), "Plan Estratégico Turismo 2012-5 y Plan de Actuaciones 2012", Fundación Turismo Valencia, Valencia.

Fundación Turismo Valencia (2017), "València Turística, hacia 2020", Fundación Turismo Valencia, Valencia.

Gui, L. y Russo, A. P. (2011): Cruise ports: a strategic nexus between regions and global lines-evidence from the Mediterranean, Maritime Policy $\mathcal{G}$ Management, 38(2),129-150, doi: 10.1080/03088839.2011.556678 
Grinberger, A. Y., Shoval, N. y McKercher, B. (2014): Typologies of tourists time-space consumption: a new approach using GPS data and GIS tools, Tourism Geographies, 16(1), 105-123, doi: $10.1080 / 14616688.2013 .869249$

Hall, C. M. (2005): Time, space, tourism and social physics. Tourism Recreation Research, 30(1), 93-98. Hall, C. M., \& Ram, Y. (2019): Measuring the Relationship between Tourism and Walkability?: Walk Score and English Tourist Attractions. Journal of Sustainable Tourism, 27(2), 223-240.

Hägerstrand, T. (1970): What about people in regional science?, Papers in Regional Science, 24(1), 7-24. Ivars, J., Celdrán. M., Triviño, A. y Vera-Rebollo, F. (2016): Tourist mobility at coastal mass destinations: implications for sustainability, WIT Transactions on Ecology and the Environment, 201, 127137, doi: $10.2495 /$ ST160111

Jaakson, R. (2004): Beyond the tourist bubble? Cruise ship passengers in port, Annals of Tourism Research, 31(1), 44-60, doi: 10.1016/j.annals.2003.08.003

Kitchin, R. (2014): The real-time city? Big data and smart urbanism, Geojournal, 79 (1), 1-14, doi: 10.1007/s10708-013-9516-8

Lau, G. y McKercher, B. (2006): Understanding Tourist Movement Patterns in a Destination: A GIS Approach, Tourism and Hospitality Research, 7, 39-49.

Leiper, N. (1979): The framework of tourism: towards a definition of tourism, tourist, and the tourist industry, Annals of Tourism Research, 6, 390-407, doi: 10.1016/0160-7383(79)90003-3

Lew, A. y McKercher, B. (2006): Modeling tourist movements: A local destination analysis, Annals of Tourism Research, 33(2), 403-423, doi: 10.1016/j.annals.2005.12.002

Li, Y., Yang, L., Shen, H. y Wu, Z. (2019): Modeling intra-destination travel behavior of tourists through spatio-temporal analysis, Journal of Destination Marketing and Management, 11, 260-269, doi: $10.1016 / j . j d m m .2018 .05 .002$

Lopes, M. J. y Dredge, D. (2018): Cruise Tourism Shore Excursions: Value for Destinations?, Tourism Planning E Development, 15 (6), 633-652, doi: 10.1080/21568316.2017.1366358

Martí, P., Serrano-Estrada, L., \& Nolasco-Cirugeda, A. (2017): Using locative social media and urban cartographies to identify and locate successful urban plazas. Cities, 64, 66-78.

https://doi.org/10.1016/j.cities.2017.02.007

McKercher, B. y Lau, G. (2008): Movement patterns of tourists within a destination, Tourism Geographies, 10(3), 355-374, doi:10.1080/14616680802236352

McKercher, B., Wong, C., \& Lau, W.C. G. (2006): How tourists consume a destination. Journal of Business Research, 59(5), 647-652.

McKercher, B. y Zoltan, J. (2014): Tourist Flows And Spatial Behavior, en Lew, A., Hall, C.M. y Williams, A. (eds.), The Wiley-Blackwell Companion to Tourism, Oxford: Wiley Blackwell, 33-44.

Md Khairi, N. D., \& Ismail, H. N. (2015): Acknowledging the Tourist Spatial Behavior for Space Management in Urban Heritage Destination. International Journal of Built Environment and Sustainability, 2(4), 317-323.

MedCruise. (2019): Cruise Activities in MedCruise Ports STATISTICS 2018. Retrieved from www.medcruise.com 
Modsching, M., Kramer, R., Gretzel, U., \& Hagen, K. (2006): Capturing the beaten paths: A novel method for analysing tourists' spatial behaviour at an urban destination. Information and Communication Technologies in Tourism 2006, 75-86.

Navarro-Ruiz, Casado-Díaz e Ivars-Baidal; (2019): Cruise Tourism: Therole of shore excursions in the overcrowding of cities, International Journal of Tourism Cities, 6(1), 197-214.

Pearce, D. (1988): Tourist time-budget. Annals of Tourism Research, 15(1), 106-121.

Pearce, D. (1995): Tourism Today. A Geographical Analysis, Harlow: Longman, $2^{\mathrm{a}}$ edición.

Prideaux, B. (2000): The role of the transport system in destination development. Tourism Management, 21(1), 53-63.

Puche-Ruiz, M. y Obiol-Menero, E. (2011): Procesos de "re-imageneering" turístico: el eclipse de la identidad local de Valencia, Cuadernos de Turismo, 28, 191-214.

Puertos del Estado (2019): «Estadística mensual», <http://www.puertos.es/es-es/estadisticas/Paginas/ estadistica_mensual.aspx $>$ (consulta: 2/5/2019).

Ram, Y. \& Hall, C. M. (2018): Walk Score and tourist accommodation. International Journal of Tourism Cities, 4(3), 369-375.

Rausell-Köster, P., Pardo-García, C., Coll-Serrano, V., \& Bustamante-Yábar, D. P. (2016): Cultural dimension and tourist satisfaction: the case of Valencia (Spain). Some notes for European urban tourism. 19th International Conference on Cultural Economics2. Valladolid: Association for Cultural Economics International.

Rodrigue, J. P. y Notteboom, T. (2013): The geography of cruises: Itineraries, not destinations, Applied Geography, 38(1), 31-42, doi: 10.1016/j.apgeog.2012.11.011

Salom-Carrasco, J. y Pitarch-Garrido, M. (2017): Análisis del impacto en el Turismo de la Estrategia de Desarrollo Urbano basada en Megaproyectos. El caso de la ciudad de Valencia, Cuadernos de Turismo, 40, pp. 573-598.

Sanz-Blas, S. y Buzova, D. (2016): Guided Tour Influence on Cruise Tourist Experience in a Port of Call: An eWOM and Questionnaire Based Approach, International Journal of Tourism Research, 18 (6), 558-566, doi: 10.1002/jtr.2073

Shoval, N. (2008): Tracking Technologies and Urban Analysis, Cities, 25, 21-28, doi: 10.1016/j.cities.2007.07.005

Shoval, N., e Isaacson, M. (2010): Tourist Mobility and Advanced Tracking Technologies, Londres: Routledge.

Tashakkori, A., \& Teddlie, C. (2003): Handbook of Mixed Methods in Social $\mathcal{E}$ Behavioral Research. SAGE Publications.

Thornton, P. R., Williams, A. M., \& Shaw, G. (1997): Revisiting time - space diaries: an exploratory case study of tourist behaviour in Cornwall, England. Environment and Planning A, 29(10), 18471867.

Van der Knaap, W (1997): The tourist's drivers: GIS Oriented Methods for Analysing Tourist Recreation Complexes. Wageningen Agricultural University. 
Van der Knaap, W. (1999): Research report: GIS oriented analysis of tourist time-space patterns to support sustainable tourism development, Tourism Geographies, 1 (1), 56-69, doi: $10.1080 / 14616689908721294$

Veal, A. J. (2006): Research Methods for Leisure and Tourism: a practical guide (3rd ed.). Harlow: Pearson Education Limited.

Vich, G., Marquet, O., \& Miralles-Guasch, C. (2019): Green exposure of walking routes and residential areas using smartphone tracking data and GIS in a Mediterranean city. Urban Forestry and Urban Greening, 40(August 2018), 275-285.

Vogel, M.P. (2011): Monopolies at sea: the role of onboard sales for the cruise industry's growth and profitability, en A. Matias, P. Nijkamp y M. Sarmento (eds.), Tourism economics: Impact analysis, Heidelberg: Physica, 211-229.

Xia, J. C., Zeephongsekul, P., \& Packer, D. (2011): Spatial and temporal modelling of tourist movements using Semi-Markov processes. Tourism Management, 32(4), 844-851.

Cómo citar este artículo:

Navarro-Ruiz, S., Ivars-Baidal, J. A., Casado-Díaz, A. B. (2020). Análisis del comportamiento espacio-temporal intradestino mediante GPS: Una aproximación de los visitantes independientes de crucero en la ciudad de València. Cuadernos de Geografía, 104, 23-42.

https://doi.org/10.7203/cguv.104.16577

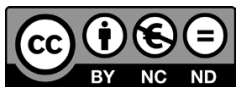

Este obra está bajo una licencia de Creative Commons Reconocimiento-NoComercial-SinObraDerivada 4.0 Internacional. 